\title{
Treatment of Posttraumatic Stress Disorder Alleviates Tinnitus-related Distress Among Veterans: a Pilot Study
}

John Moring ( $\nabla$ moringj@uthscsa.edu )

Department of Psychiatry and Behavioral Sciences, University of Texas Health Science Center at San Antonio, $7550 \mathrm{IH}-10$ West, Suite 1325, San Antonio, TX 78229, USA https://orcid.org/0000-0001-58103801

\section{Patricia Resick}

Duke University Medical Center

\section{Alan Peterson}

University of Texas Health Science Center at San Antonio

\section{Fatima Husain}

University of Illinois at Urbana-Champaign

\section{Carlos Esquivel}

Wilford Hall Ambulatory Surgical Center

\section{Stacey Young-McCaughan}

University of Texas Health Science Center at San Antonio

\section{Elsa Granato}

Wilford Hall Ambulatory Surgical Center

\section{Peter Fox}

University of Texas Health Science Center at San Antonio

\section{Research}

Keywords: Tinnitus, Posttraumatic Stress Disorder, Veterans, Military, Cognitive Processing Therapy, Treatment, Clinical Trial

Posted Date: October 22nd, 2020

DOI: https://doi.org/10.21203/rs.3.rs-95013/v1

License: (c) (i) This work is licensed under a Creative Commons Attribution 4.0 International License. Read Full License 


\section{Abstract}

Background: Military service personnel are at increased risk for developing tinnitus due to heightened exposure to acoustic trauma. The auditory disorder is a leading service-connected disability among veterans and is highly comorbidly diagnosed with posttraumatic stress disorder (PTSD). The biopsychosocial model illustrates that chronic health conditions are exacerbated or maintained by psychiatric distress. Therefore, alleviation of such psychiatric distress can have beneficial impacts on health conditions, such as tinnitus. The aim of this study was to determine whether individuals with both disorders who receive evidence-based therapy for PTSD, will experience decreases in both PTSD and tinnitus-related distress.

Methods: Veterans with comorbid bothersome tinnitus and PTSD received Cognitive Processing Therapy (CPT) and were assessed for PTSD, tinnitus-related distress, and depression at baseline and one month post-treatment follow-up.

Results: At post-treatment follow-up, participants demonstrated significant decreases in PTSD symptoms compared to their baseline scores. Participants also demonstrated decreased tinnitus-related distress and depression, with high effect sizes.

Discussion: This pilot study demonstrated the benefit of addressing psychiatric distress, such as PTSD, to simultaneously target bothersome tinnitus. Although not statistically significant due to the small sample size, large effect sizes indicate that tinnitus-related distress decreased as a function of receiving evidence-based therapy for PTSD. Future clinical trials should increase sample sizes and compare effects to control conditions.

\section{Trial Registration}

ClinicalTrials.gov. NCT03702166. Registered October 10, 2018, https://clinicaltrials.gov/ct2/show/NCT03702166? cond=tinnitus\&cntry=US\&state=US\%3ATX\&city=San+Antonio\&draw=1\&rank=1

\section{Background}

Posttraumatic stress disorder (PTSD) is characterized as a psychological reaction to actual or threatened death, serious injury, or sexual violence [1]. Over $90 \%$ of service members and veterans deployed post9/11 have been exposed to at least one type of traumatic event during their deployments [2] and up to $32 \%$ of military service members and veterans have been diagnosed with PTSD [3, 4]. Comorbid health conditions, such as headaches, traumatic brain injury (TBI), and depression have been shown to worsen overall PTSD symptom presentation [5]. Another common co-occurring disorder with PTSD is tinnitus, but it is unknown how it affects PTSD symptom presentation. 
Tinnitus is defined as a phantom auditory perception, in one or both ears, without an objective noise source [6]. Types of tinnitus percept include ringing, buzzing, or a rushing sound; the intensity frequency and duration of these percepts vary among individuals [7]. Tinnitus-related distress includes the cognitive, emotional, and behavioral symptomatic sequalae related to the experience of chronic tinnitus. The symptom clusters of tinnitus-related distress are heterogeneous and affects cognitive and emotional wellbeing $[8,9]$ and can include symptoms of anxiety and depression $[10]$.

Military personnel have a much higher risk of developing tinnitus due to significant exposure to acoustic trauma through proximity to military aircraft [11], weapon use [12], and combat-related hazards such as car bombs, improvised explosive devices (IEDs), incoming mortar attacks, and rocket-propelled grenades [13]. Exposure to acoustic trauma increases the likelihood of developing tinnitus before, during, and after deployment [14]. Although the defining symptoms of tinnitus (an illusory auditory percept) and PTSD (a trauma disorder) are distinct, their prevalence rates indicate a possible connection between the two disorders.

First, a chart review of veterans treated over a four-year period indicated that $34 \%$ of veterans with tinnitus also carried a PTSD diagnosis [15]. Upon closer examination, tinnitus-related distress and PTSD may reflect common sources and/or symptoms. The same psychologically traumatic event could contribute to both PTSD and tinnitus (e.g., IEDs, car bomb). Tinnitus can be conceptualized as a conditioned stimulus, reminding the individual of the traumatic event (Criterion B of PTSD) [1]. In a study of Cambodian refugees with PTSD symptoms, an overwhelming $92 \%$ of that cohort reported that flashbacks were accompanied by tinnitus [16], suggesting the relationship between tinnitus and reexperiencing symptoms of PTSD. The same study demonstrated that tinnitus-associated negative mood and cognitive symptoms also predicted PTSD severity. Other studies have reported heightened symptoms of avoidance [15], hypervigilance, and hyperarousal [17] among tinnitus patients, representing Criterion $C$ and $E$ of PTSD, respectively.

There is strong evidence to support involvement of a shared neurobiological mechanism between tinnitus and PTSD. The auditory vigilance network (A1/VAN) is highly implicated among PTSD patients [18]. In fact, the auditory network contains the most regions that differentiate veterans with PTSD versus combat-exposed veterans and civilians without PTSD [19]. The A1/VAN is also the most frequently implicated network in patients with tinnitus [20-22]. Another study found dysregulated connectivity within the A1/VAN, and higher connectivity between the A1/VAN and the visual cortex among PTSD patients [18]. These findings suggest that the genesis and maintenance of tinnitus and PTSD may involve similar neurobiological substrates and/or processes.

Other studies point to exacerbation of tinnitus-related distress as a function of psychiatric distress. Between $32 \%$ and $45 \%$ of individuals with tinnitus also suffer from anxiety disorders [23], indicating a potentially strong psychiatric component of tinnitus-related distress, either as a premorbid factor or with tinnitus onset. The same study showed that $40 \%$ of individuals with tinnitus also reported a family history of psychiatric disorders. Functional outcomes for individuals with both tinnitus and PTSD are also 
significantly worse than for those with tinnitus only, or tinnitus and any other psychological disorder [17]. This finding suggests especially deleterious outcomes when tinnitus co-occurs with PTSD. Investigation of innovative approaches to the simultaneous management of both conditions is warranted.

Current models borrowed from the chronic pain literature suggest that functional outcomes can be improved when psychological factors are targeted. The biopsychosocial model $[24,25]$ incorporates biomedical factors, behaviors, emotions, cognitions, cultural values, social support, and socioeconomic status as factors to more fully understand the origins of pain. Framed within the biopsychosocial model, the fear-avoidance model describes a cycle initiated by pain, followed by a constellation of cognitive, emotional, and behavioral responses that may reinforce pain and disability [26, 27]. A key component of the fear-avoidance model is its identification and restructuring of maladaptive cognitions related to the pain or to the implications of painful sensations. Catastrophic cognition, for example, can lead to a cascade of consequences, including overestimation of future pain, avoidance of activities, and physical deconditioning, resulting in reduced levels of function [28]. The fear-avoidance model emphasizes the connection between psychiatric distress and chronic pain, which is further supported by neurobiological [29] and psychological studies [30-32] studies. It is likely that PTSD, tinnitus, and chronic pain all manifest central nervous system changes that persist long after original external or peripheral trauma. It is reasonable to consider that evidence-based therapies for one of these conditions (e.g., PTSD) may also serve to reduce distress associated with another (e.g., tinnitus).

Cognitive processing therapy [33] is one such evidence-based psychotherapy for PTSD. Studies have demonstrated that CPT is efficacious among childhood sexual and physical abuse survivors [34, 35], female sexual assault survivors [36] and military service personnel and veterans [37-41]. Meta-analyses have also shown that CPT has the largest mean effect size compared to any other treatment for PTSD [42-44]. CPT usually consists of 12 sessions, each lasting 50-60 minutes. Patients learn how to recognize dysfunctional thoughts ("stuck points") that are related to the traumatic experience and larger themes of safety, trust, power and control, esteem, and intimacy. Moving forward in therapy, patients challenge these stuck points and learn how to develop more realistic thoughts, acceptance of the traumatic event, and experiencing natural emotions associated with the trauma.

The alleviation of PTSD may serve to decrease tinnitus-related distress in two ways. First, since tinnitusrelated distress and PTSD share common symptoms [15-17], reduction of avoidance, improved emotional and cognitive flexibility, and reduced reactivity may extend to reduce tinnitus-related dysfunction. Additionally, according to the biopsychosocial and fear-avoidance models, PTSD patients who change their dysfunctional beliefs and learn to experience more positive emotions through evidencebased psychotherapy may also benefit by reduced tinnitus-related distress.

The current study investigated the relationship between PTSD-related psychiatric distress and tinnitusrelated distress. Based on the reasoning of the biopsychosocial and fear-avoidance models, we expected that negative emotional, cognitive, and behavioral states associated with PTSD serve to exacerbate tinnitus-related distress. Therefore, it was hypothesized that from baseline to one month post-treatment 
follow-up, individuals with PTSD and bothersome tinnitus who received CPT would experience decreased PTSD symptoms as well as decreased tinnitus-related distress.

\section{Methods}

\section{Participants}

Informed consent was obtained from all individual participants included in the study. Participants were U.S. veterans, 18 years of age or older who sought treatment for PTSD. Inclusion criteria required the experience of a Criterion A trauma defined by the DSM-5 [1], a diagnosis of PTSD from an independent evaluator using the Clinician-Administered PTSD Scale for DSM-5 (CAPS-5) [45], and self-reported bothersome tinnitus, operationalized as a score of 34 or higher on the Tinnitus Functional Index (TFI) [46]. English literacy and stability on psychotropic medications was also required. Exclusion criteria included suicide or homicide risk warranting immediate intervention, significant alcohol and/or substance abuse that would prevent participants from attending therapy or completing practice assignments, active psychosis, and/or severe traumatic brain injury (TBI).

This study was reviewed by the University of Texas Health Science Center at San Antonio Institutional Review Board (Protocol \#HSC20180524H). All procedures performed in studies involving human participants were in accordance with the ethical standards of the institution and with the 1964 Helsinki declaration and its later amendments or comparable ethical standards. For each session and evaluation, adverse events were monitored using an AE-monitoring form used in previous studies [47].

\section{Measures}

Clinician-Administered PTSD Scale for DSM-5(CAPS-5) [45]. The CAPS-5 is a semi-structured interview that measures the DSM-5 [1] symptoms of PTSD; to reach diagnostic threshold, this scale requires the presence of at least one intrusion symptom, one avoidance symptom, two cognition or mood symptoms, and two arousal symptoms for a period of one month or more. There are 20 symptom items and responses that are rated on a 5-point scale ranging from 0 (absent) to 4 (extreme/incapacitating); a total PTSD symptom severity score ranges from $0-80$ as the sum of the 20 symptom items.

Patient Health Questionnaire-9 (PHQ-9) [48]. The PHQ-9 is a 9-item self-report measure that measures the severity of affective and somatic symptoms related to depression and depressive disorders. Respondents rate the frequency with which they have been bothered by depressive symptoms within the past two weeks on a scale from 0 ("not at all") to 3 ("nearly every day"). The PHQ-9 has high internal consistency and correlates strongly with other measures of depression.

Tinnitus Functional Index (TFI) [49]. The TFI is used specifically for clinical trials in which tinnitusassociated symptom severity may change over time. The TFI is comprised of 25 questions forming 8 subscales. Subscales include intrusiveness of tinnitus, sense of control, cognitive interference, sleep 
disturbance, auditory issues, relaxation issues, quality of life, and emotional distress. Individuals with TFI scores of below 25 are classified as "mild tinnitus," while scores between 25 and 50 are considered "significant problems," and scores above 50 indicate severe tinnitus that warrants more intensive therapeutic approaches. Additionally, reductions of approximately 14 points on the TFI represent clinically meaningful change [49]. The TFI was used in this study as a screener to ensure that enrolled participants had bothersome tinnitus, defined as a score of 34 or higher.

PTSD Checklist for DSM-5 (PCL-5) [50]. The PCL-5 has excellent psychometric characteristics for screening and as a secondary indicator of PTSD symptom severity [51]. The PCL-5 is a 20-item self-report measure, selected for its dimensional sensitivity; higher scores reflect greater PTSD severity.

\section{Procedures}

Participants were recruited from advertisements on social media and by direct referrals from Department of Veterans Affairs (VA) providers. All participants met criteria for PTSD and tinnitus during baseline assessment conducted by an independent evaluator who was blinded from the treatment condition and assessment timepoint. Baseline measures of PTSD (CAPS-5), depression (PHQ-9), and tinnitus-related distress (TFI) were gathered.

If participants were found eligible and provided consent, they met with the study therapist (JCM) prior to beginning CPT. This initial session focused on gathering psychosocial information, a review of the participant's index traumatic event, discussion of treatment goals and expectations of therapy, and creation of a therapy schedule. Throughout CPT, participants completed the PCL-5 (even numbered sessions) and the PHQ-9 and TFI (odd numbered sessions). Upon completion of CPT, participants were scheduled for a one-month follow-up to assess for PTSD, depression, and tinnitus-related distress.

\section{Data Analytic Strategy}

Primary variables of interest to this study included change scores of the CAPS-5, PHQ-9, and TFI, from baseline to post-treatment. Multiple t-tests were conducted with Bonferroni correction for multiple comparisons. Alpha level for significance, with three tests, was set ata $=.017$.

\section{Results}

Participants were 10 veterans diagnosed with PTSD and bothersome tinnitus. Table 1 shows participants' demographic information. The mean age of participants was 45.3 years, with an average of 15.74 years of service in the military. One participant (10\%) dropped out of the study after session 4 of CPT, and 1 participant did not complete post-treatment assessments.

Multiple t-tests, with Bonferroni corrections for multiple comparisons, showed significant decreased symptoms of PTSD as measured by the CAPS- 5 from pre-treatment $(M=33.13, S D=.8 .08)$ to post- 
treatment $(M=16.13, S D=14.45), t(1,7)=3.42, p=.01, d=1.29$. Decreases in depression from pretreatment $(M=14.10, S D=6.59)$ to post-treatment $(M=7.38, S D=4.98), t(1,7)=2.68, p=.031$, and tinnitus-related distress from pre-treatment $(M=65.16, S D=15.97)$ to post-treatment $(M=41.95, S D=$ $24.42), t(1,7)=2.56, p=.038$, approached significance. Cohen's $d$ effect sizes for depression and tinnitus-related distress were 1.01 and .97, respectively. Figure 1 shows pre- and post-treatment scores.

Figure 2 shows session-by-session mean PCL-5, PHQ-9, and TFI scores. The mean change on the TFI was $M=22.34(S D=24.71)$, and half $(N=4)$ of the participants experienced a clinically meaningful change on the $\mathrm{TFI}$, with reductions greater than 14 points [49].

\section{Discussion}

Tinnitus and PTSD are the first and third most common service-connected disabilities, respectively, in U.S. military veterans. The development and prevalence of these conditions will likely increase over time among veterans who served post-9/11 deployments to Iraqi, Afghanistan, and surrounding locations. During deployment, service members are at higher risk of experiencing acoustic trauma [14] and psychological trauma [52]. There is a continuing need for evidence-based PTSD and tinnitus treatments that can improve veterans' level of functioning and quality of life.

Based on prior research and existing models of pain and psychiatric distress, it was hypothesized that treatment for PTSD symptoms would also reduce tinnitus-related distress. Research on the prevalence, functional outcomes, and neurobiological basis of these conditions indicate a possible connection. Shared symptoms include flashbacks when tinnitus reminds individuals of their psychological trauma [16], avoidance and aversions to loud signals [15], exaggerated and erroneous beliefs [16], and hyperarousal [17]. Biopsychosocial and fear-avoidance models account for the role of cognitions and emotion in disability status among chronic pain patients. More specifically, Marshall et al. [53] showed that the relationship between pain and disability was significantly mediated by fear, catastrophic thinking, and depression for individuals who were active on a weekly basis. Emotional and psychiatric distress appears to have a negative impact on pain and on functional outcomes related to pain. Therefore, we hypothesized that alleviation of PTSD-related psychiatric distress, through CPT, would also help to alleviate depression-, and tinnitus-related distress.

Results of this study partially supported its hypothesis. A total of 10 participants with comorbid bothersome tinnitus and PTSD completed CPT [33]. At a one-month post-treatment follow-up, participants showed a significant reduction in their PTSD symptoms, with a large effect size. Though not statistically significant, participants also experienced decreases in symptoms of depression and tinnitusrelated distress, with large effect sizes. This study demonstrated promising results for the possible alleviation of tinnitus through evidence-based psychotherapy for PTSD.

Limitations of this study include its small sample size, which reduced its statistical power and limited the significance of pre- to post-treatment changes in depression and tinnitus-related distress. The Cohen's $d$ effect sizes of all analyses were large, indicating that these changes likely would have reached 
significance in a larger cohort. Other limitations of the study include the lack of audiometric evaluation to characterize participants' auditory function more fully as it relates to tinnitus. Future studies should include larger participant sets and more detailed evaluation of tinnitus and hearing status; a randomized clinical trial to include and compare other treatments would be informative.

\section{Conclusions}

In summary, the hypotheses for the current study were partially supported. Through CPT, participants learned how to recognize and challenge maladaptive thoughts related to trauma and present-day difficulties, and to generate more balanced thoughts to reduce their PTSD symptom. Attenuation of their tinnitus-related distress was also observed, though this change did not reach statistical significance. Future, larger studies should examine the neurobiological relationship between tinnitus and PTSD, and consider approaches to more specifically tailor psychotherapy, compare alternative treatment strategies, or develop innovative neuro-modulatory therapies.

\section{Abbreviations}

\section{A1/VAN}

Auditory Vigilance Attention Network; CAPS-5:Clinician-Administered PTSD Scale for DSM-5; CPT:Cognitive Processing Therapy; DSM-5:Diagnostic and Statistical Manual of Mental Disorders, 5th Edition; IED:Improvised Explosive Device; PCL-5:PTSD Checklist for DSM-5; PHQ-9:Patient Health Questionnaire-9; PTSD:Posttraumatic Stress Disorder; TFI:Tinnitus Functional Index

\section{Declarations}

\section{Ethics approval and consent to participate}

All study procedures were approved and monitored by the Institutional Review Board at the University of Texas Health Science Center at San Antonio. The study was conducted in accordance with the Declaration of Helsinki. All study participants provided written informed consent prior to any study procedure.

\section{Consent for publication}

Not applicable.

\section{Availability of supporting data}

Final data sets from this pilot study will be shared in accordance with a Data Use Agreement that prohibits the recipient from identifying or re-identifying any individual whose data are included in the dataset. We will make sufficient data and descriptors available to confirm conclusions in the publication, run duplicate statistical analyses, and perform additional analyses. 


\section{Competing interests}

The authors declare that they have no competing interests.

\section{Funding}

The project described was supported by the National Center for Advancing Translational Sciences, National Institutes of Health, through the Clinical and Translational Science Award (CTSA) KL2 TR002646. The content is solely the responsibility of the authors and does not necessarily represent the official views of the National Institutes of Health, Department of Veterans Affairs, Department of Defense, or the U.S. Government.

\section{Authors' contributions}

JCM, PAR, ALP, FTM, CE, and PF assisted with the design, writing, and interpretation of results. SY-M assisted with writing and regulatory aspects of the study. EG assisted with participant recruitment and study design.

\section{Acknowledgements}

We thank Amanda Flores, Antoinette Brundige, and Deanne Hargita for administrative support.

\section{Authors' information}

Patricia A. Resick (patricia.resick@duke.edu); Alan L. Peterson (petersona3@uthscsa.edu); Fatima T. Husain (husainf@acnlab.com); Carlos Esquivel (carlos.r.esquivel.civ@mail.mil); Stacey YoungMcCaughan (youngs1@uthscsa.edu); Elsa Granato (elsa.c.granato.ctr@mail.mil); Peter Fox (fox@uthscsa.edu)

\section{References}

1. American Psychiatric Association. Diagnostic and statistical manual of mental disorders : DSM-5 [text]. Arlington, VA: American Psychiatric Association;; 2013.

2. Tanielian TL, Jaycox L, Rand Corporation. Invisible wounds of war : psychological and cognitive injuries, their consequences, and services to assist recovery. Santa Monica, CA: RAND; 2008.

3. Hoge CW, Auchterlonie JL, Milliken CS. Mental health problems, use of mental health services, and attrition from military service after returning from deployment to Iraq or Afghanistan. JAMA. 2006;295(9):1023-32. doi:10.1001/jama.295.9.1023

4. Gaylord KM, Cooper DB, Mercado JM, Kennedy JE, Yoder LH, Holcomb JB. Incidence of posttraumatic stress disorder and mild traumatic brain injury in burned service members: preliminary report. J. Trauma. 2008;64(2 Suppl):S200-5; discussion S5-6. doi:10.1097/TA.0b013e318160ba42 
5. Wilk JE, Herrell RK, Wynn GH, Riviere LA, Hoge CW. Mild traumatic brain injury (concussion), posttraumatic stress disorder, and depression in U.S. soldiers involved in combat deployments: association with postdeployment symptoms. Psychosom. Med. 2012;74(3):249-57. doi:10.1097/PSY.0b013e318244c604

6. Hazell JW, Jastreboff PJ. Tinnitus. I: Auditory mechanisms: a model for tinnitus and hearing impairment. J. Otolaryngol. 1990;19(1):1-5.

7. Unterrainer J, Greimel KV, Leibetseder M, Koller T. Experiencing tinnitus: which factors are important for perceived severity of the symptom? Int. Tinnitus J. 2003;9(2):130-3.

8. Tegg-Quinn S, Bennett RJ, Eikelboom RH, Baguley DM. The impact of tinnitus upon cognition in adults: A systematic review. Int. J. Audiol. 2016;55(10):533-40.

doi:10.1080/14992027.2016.1185168

9. Trevis KJ, McLachlan NM, Wilson SJ. A systematic review and meta-analysis of psychological functioning in chronic tinnitus. Clin. Psychol. Rev. 2018;60:62-86. doi:10.1016/j.cpr.2017.12.006

10. Hesser $\mathrm{H}$, Andersson $\mathrm{G}$. The role of anxiety sensitivity and behavioral avoidance in tinnitus disability. Int. J. Audiol. 2009;48(5):295-9. doi:10.1080/14992020802635325

11. Smalt CJ, Lacirignola J, Davis SK, Calamia PT, Collins PP. Noise dosimetry for tactical environments. Hear. Res. 2017;349:42-54. doi:10.1016/j.heares.2016.11.008

12. Beach EF, Gilliver M, Williams W. Leisure noise exposure: participation trends, symptoms of hearing damage, and perception of risk. Int. J. Audiol. 2013;52 Suppl 1:S20-5.

doi:10.3109/14992027.2012.743050

13. Moring JC, Peterson AL, Kanzler KE. Tinnitus, Traumatic Brain Injury, and Posttraumatic Stress Disorder in the Military. Int. J. Behav. Med. 2018;25(3):312-21. doi:10.1007/s12529-017-9702-z

14. Yurgil KA, Clifford RE, Risbrough VB, et al. Prospective Associations Between Traumatic Brain Injury and Postdeployment Tinnitus in Active-Duty Marines. J. Head Trauma Rehabil. 2016;31(1):30-9. doi:10.1097/HTR.0000000000000117

15. Fagelson MA. The association between tinnitus and posttraumatic stress disorder. Am J Audiol. 2007;16(2):107-17. doi:10.1044/1059-0889(2007/015)

16. Hinton DE, Chhean D, Pich V, Hofmann SG, Barlow DH. Tinnitus among Cambodian refugees: relationship to PTSD severity. J. Trauma. Stress. 2006;19(4):541-6. doi:10.1002/jts.20138

17. Fagelson MA, Smith SL. Tinnitus Self-Efficacy and Other Tinnitus Self-Report Variables in Patients With and Without Post-Traumatic Stress Disorder. Ear Hear. 2016;37(5):541-6. doi:10.1097/aud.0000000000000290

18. Shang J, Lui S, Meng Y, et al. Alterations in low-level perceptual networks related to clinical severity in PTSD after an earthquake: a resting-state fMRI study. PLoS One. 2014;9(5):e96834. doi:10.1371/journal.pone.0096834

19. Vanasse TJ, Franklin C, Salinas FS, et al. A resting-state network comparison of combat-related PTSD with combat-exposed and civilian controls. Soc. Cogn. Affect. Neurosci. 2019;14(9):933-45. doi:10.1093/scan/nsz072 
20. De Ridder D, Vanneste S, Weisz N, et al. An integrative model of auditory phantom perception: tinnitus as a unified percept of interacting separable subnetworks. Neurosci. Biobehav. Rev. 2014;44:16-32. doi:10.1016/j.neubiorev.2013.03.021

21. Husain FT. Neural networks of tinnitus in humans: Elucidating severity and habituation. Hear. Res. 2016;334:37-48. doi:10.1016/j.heares.2015.09.010

22. Lv H, Zhao P, Liu Z, et al. Abnormal regional activity and functional connectivity in resting-state brain networks associated with etiology confirmed unilateral pulsatile tinnitus in the early stage of disease. Hear. Res. 2017;346:55-61. doi:10.1016/j.heares.2017.02.004

23. Pattyn T, Van Den Eede F, Vanneste $S$, et al. Tinnitus and anxiety disorders: A review. Hear. Res. 2016;333:255-65. doi:10.1016/j.heares.2015.08.014

24. Engel GL. The clinical application of the biopsychosocial model. Am. J. Psychiatry. 1980;137(5):53544. doi:10.1176/ajp.137.5.535

25. Peterson AL, Goodie JL, \& Andrasik F. Introduction and overview of biopsychosocial assessment in clinical health psychology. In Andrasik F, Goodie JL, \& Peterson AL, editors. Biopsychosocial assessment in clinical health psychology: A handbook. New York, NY: Guilford; 2015. pp. 3-7.

26. Leeuw M, Goossens ME, Linton SJ, Crombez G, Boersma K, Vlaeyen JW. The fear-avoidance model of musculoskeletal pain: current state of scientific evidence. J. Behav. Med. 2007;30(1):77-94. doi:10.1007/s10865-006-9085-0

27. Vlaeyen JW, Linton SJ. Fear-avoidance and its consequences in chronic musculoskeletal pain: a state of the art. Pain. 2000;85(3):317-32. doi:10.1016/s0304-3959(99)00242-0

28. Crombez G, Eccleston C, Van Damme S, Vlaeyen JW, Karoly P. Fear-avoidance model of chronic pain: the next generation. Clin. J. Pain. 2012;28(6):475-83. doi:10.1097/AJP.0b013e3182385392

29. Bruehl S, Burns JW, Chung OY, Chont M. Pain-related effects of trait anger expression: neural substrates and the role of endogenous opioid mechanisms. Neurosci. Biobehav. Rev. 2009;33(3):47591. doi:10.1016/j.neubiorev.2008.12.003

30. Asmundson GJ, Coons MJ, Taylor S, Katz J. PTSD and the experience of pain: research and clinical implications of shared vulnerability and mutual maintenance models. Can. J. Psychiatry. 2002;47(10):930-7. doi:10.1177/070674370204701004

31. Davis DA, Luecken LJ, Zautra AJ. Are reports of childhood abuse related to the experience of chronic pain in adulthood? A meta-analytic review of the literature. Clin. J. Pain. 2005;21(5):398-405. doi:10.1097/01.ajp.0000149795.08746.31

32. Varinen A, Kosunen E, Mattila K, Koskela T, Sumanen M. The relationship between childhood adversities and fibromyalgia in the general population. J. Psychosom. Res. 2017;99:137-42. doi:10.1016/j.jpsychores.2017.06.011

33. Resick PA, Monson CM, Chard KM. Cognitive processing therapy for PTSD : a comprehensive manual. New York: Guilford Press; 2017.

34. Chard KM. An evaluation of cognitive processing therapy for the treatment of posttraumatic stress disorder related to childhood sexual abuse. J. Consult. Clin. Psychol. 2005;73(5):965-71. 
doi:10.1037/0022-006x.73.5.965

35. Rosner R, Rimane E, Frick U, et al. Effect of Developmentally Adapted Cognitive Processing Therapy for Youth With Symptoms of Posttraumatic Stress Disorder After Childhood Sexual and Physical Abuse: A Randomized Clinical Trial. JAMA Psychiatry. 2019;76(5):484-91.

doi:10.1001/jamapsychiatry.2018.4349

36. Resick PA, Galovski TE, Uhlmansiek MO, Scher CD, Clum GA, Young-Xu Y. A randomized clinical trial to dismantle components of cognitive processing therapy for posttraumatic stress disorder in female victims of interpersonal violence. J. Consult. Clin. Psychol. 2008;76(2):243-58. doi:10.1037/0022$006 x .76 .2 .243$

37. Kaysen D, Schumm J, Pedersen ER, Seim RW, Bedard-Gilligan M, Chard K. Cognitive processing therapy for veterans with comorbid PTSD and alcohol use disorders. Addict. Behav. 2014;39(2):4207. doi:10.1016/j.addbeh.2013.08.016

38. Monson CM, Schnurr PP, Resick PA, Friedman MJ, Young-Xu Y, Stevens SP. Cognitive processing therapy for veterans with military-related posttraumatic stress disorder. J. Consult. Clin. Psychol. 2006;74(5):898-907. doi:10.1037/0022-006X.74.5.898

39. Morland LA, Mackintosh MA, Greene CJ, et al. Cognitive processing therapy for posttraumatic stress disorder delivered to rural veterans via telemental health: a randomized noninferiority clinical trial. J. Clin. Psychiatry. 2014;75(5):470-6. doi:10.4088/JCP.13m08842

40. Resick PA, Wachen JS, Mintz J, et al. A randomized clinical trial of group cognitive processing therapy compared with group present-centered therapy for PTSD among active duty military personnel. J. Consult. Clin. Psychol. 2015;83(6):1058-68. doi:10.1037/ccp0000016

41. Resick PA, Wachen JS, Dondanville KA, et al. Effect of Group vs Individual Cognitive Processing Therapy in Active-Duty Military Seeking Treatment for Posttraumatic Stress Disorder: A Randomized Clinical Trial. JAMA Psychiatry. 2017;74(1):28-36. doi:10.1001/jamapsychiatry.2016.2729

42. Cusack K, Jonas DE, Forneris CA, et al. Psychological treatments for adults with posttraumatic stress disorder: A systematic review and meta-analysis. Clin. Psychol. Rev. 2016;43:128-41. doi:10.1016/j.cpr.2015.10.003

43. Haagen JF, Smid GE, Knipscheer JW, Kleber RJ. The efficacy of recommended treatments for veterans with PTSD: A metaregression analysis. Clin. Psychol. Rev. 2015;40:184-94. doi:10.1016/j.cpr.2015.06.008

44. Watts BV, Schnurr PP, Mayo L, Young-Xu Y, Weeks WB, Friedman MJ. Meta-analysis of the efficacy of treatments for posttraumatic stress disorder. J. Clin. Psychiatry. 2013;74(6):e541-50. doi:10.4088/JCP.12r08225

45. Weathers FW, Blake DD, Schnurr PP, Kaloupek DG, Marx BP, Keane TM. The Clinician Administered PTSD Scale for DSM-5 (CAPS-5). 2013. www.ptsd.va.gov.

46. Henry JA, Griest S, Thielman E, McMillan G, Kaelin C, Carlson KF. Tinnitus Functional Index: Development, validation, outcomes research, and clinical application. Hear. Res. 2016;334:58-64. doi:10.1016/j.heares.2015.06.004 
47. Peterson AL, Roache JD, Raj J, Young-McCaughan S, Consortium SS. The need for expanded monitoring of adverse events in behavioral health clinical trials. Contemp. Clin. Trials. 2013;34(1):152-4. doi:10.1016/j.cct.2012.10.009

48. Kroenke K, Spitzer RL, Williams JB. The PHQ-9: validity of a brief depression severity measure. J. Gen. Intern. Med. 2001;16(9):606-13. doi:10.1046/j.1525-1497.2001.016009606.x

49. Meikle MB, Henry JA, Griest SE, et al. The tinnitus functional index: development of a new clinical measure for chronic, intrusive tinnitus. Ear Hear. 2012;33(2):153-76.

doi:10.1097/AUD.0b013e31822f67c0

50. Weathers FW, Litz BT, Keane TM, Palmieri PA, Marx BP, Schnurr PP. The PTSD Checklist for DSM-5 (PCL-5). 2013. www.ptsd.va.gov.

51. McDonald SD, Calhoun PS. The diagnostic accuracy of the PTSD checklist: a critical review. Clin. Psychol. Rev. 2010;30(8):976-87. doi:10.1016/j.cpr.2010.06.012

52. Hoge CW, Castro CA, Messer SC, McGurk D, Cotting DI, Koffman RL. Combat duty in Iraq and Afghanistan, mental health problems, and barriers to care. N. Engl. J. Med. 2004;351(1):13-22. doi:10.1056/NEJMoa040603

53. Marshall PWM, Schabrun S, Knox MF. Physical activity and the mediating effect of fear, depression, anxiety, and catastrophizing on pain related disability in people with chronic low back pain. PLoS One. 2017;12(7):e0180788. doi:10.1371/journal.pone.0180788

\section{Tables}

Table 1. Demographic Characteristics 


\begin{tabular}{|ll|}
\hline Characteristic & Total Sample $(N=10)$ \\
\hline Age & $45.3(\mathrm{SD}, 9.75)$ \\
\hline Male & $9(90 \%)$ \\
\hline Married & $7(70 \%)$ \\
\hline Ethnicity & \\
\hline Hispanic & $4(40 \%)$ \\
\hline Caucasian/Non-Hispanic & $4(40 \%)$ \\
\hline African American & $2(20 \%)$ \\
\hline Education & \\
\hline Associates degree & $2(20 \%)$ \\
\hline College/Graduate degree & $8(80 \%)$ \\
\hline Branch & \\
\hline Army & $8(80 \%)$ \\
\hline Airforce & $1(10 \%)$ \\
\hline Marines & $1(10 \%)$ \\
\hline Enlisted Rank & $9(90 \%)$ \\
\hline Months in Military & $188.9(S D, 105.75)$ \\
\hline Typical Duty & \\
\hline Combat Arms & $5(50 \%)$ \\
\hline Combat Support & $2(20 \%)$ \\
\hline Combat Service Support & $3(30 \%)$ \\
\hline
\end{tabular}

Figures 


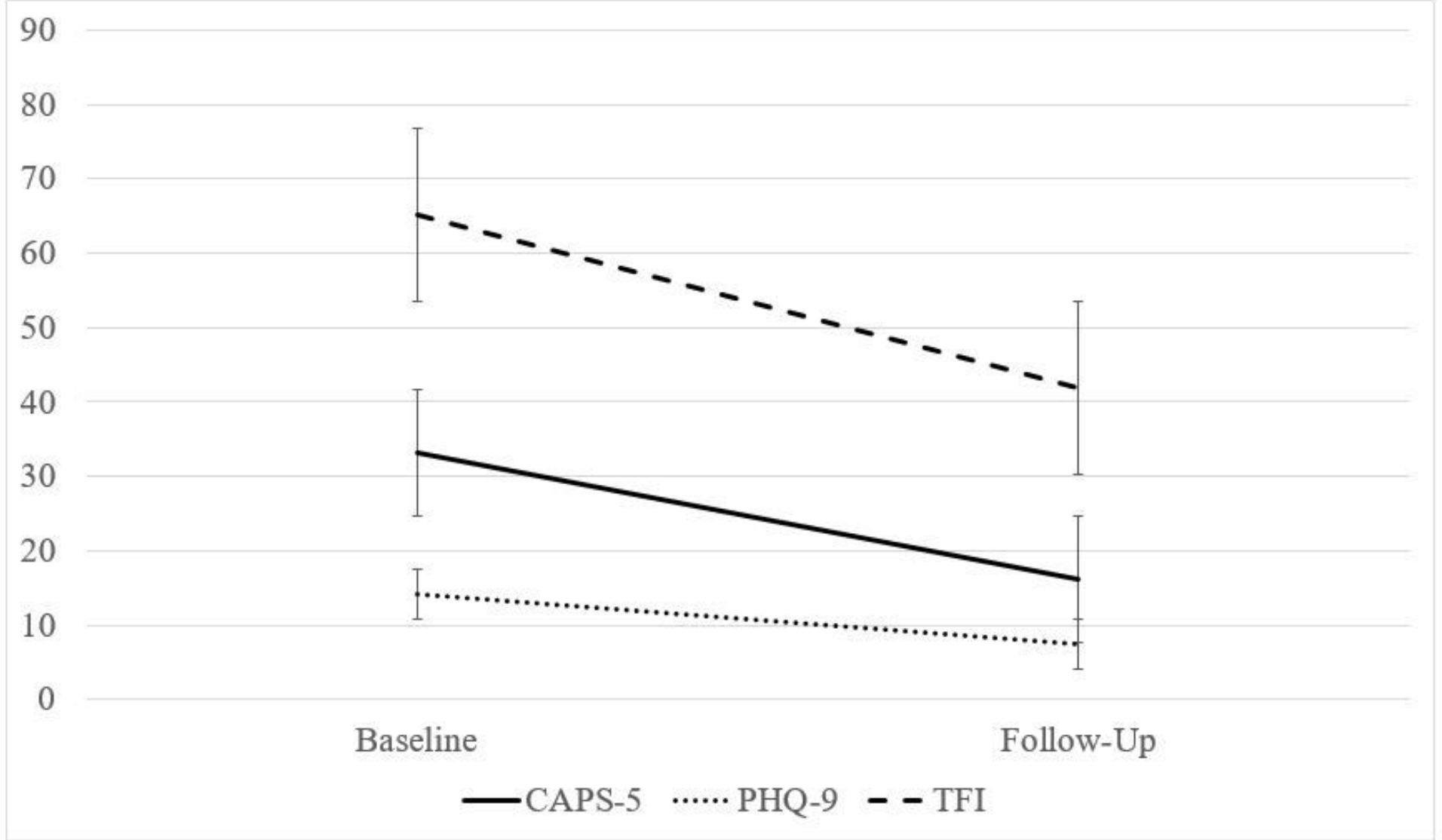

Figure 1

Pre-Post Treatment Scores of the CAPS-5, TFI, and PHQ-9 


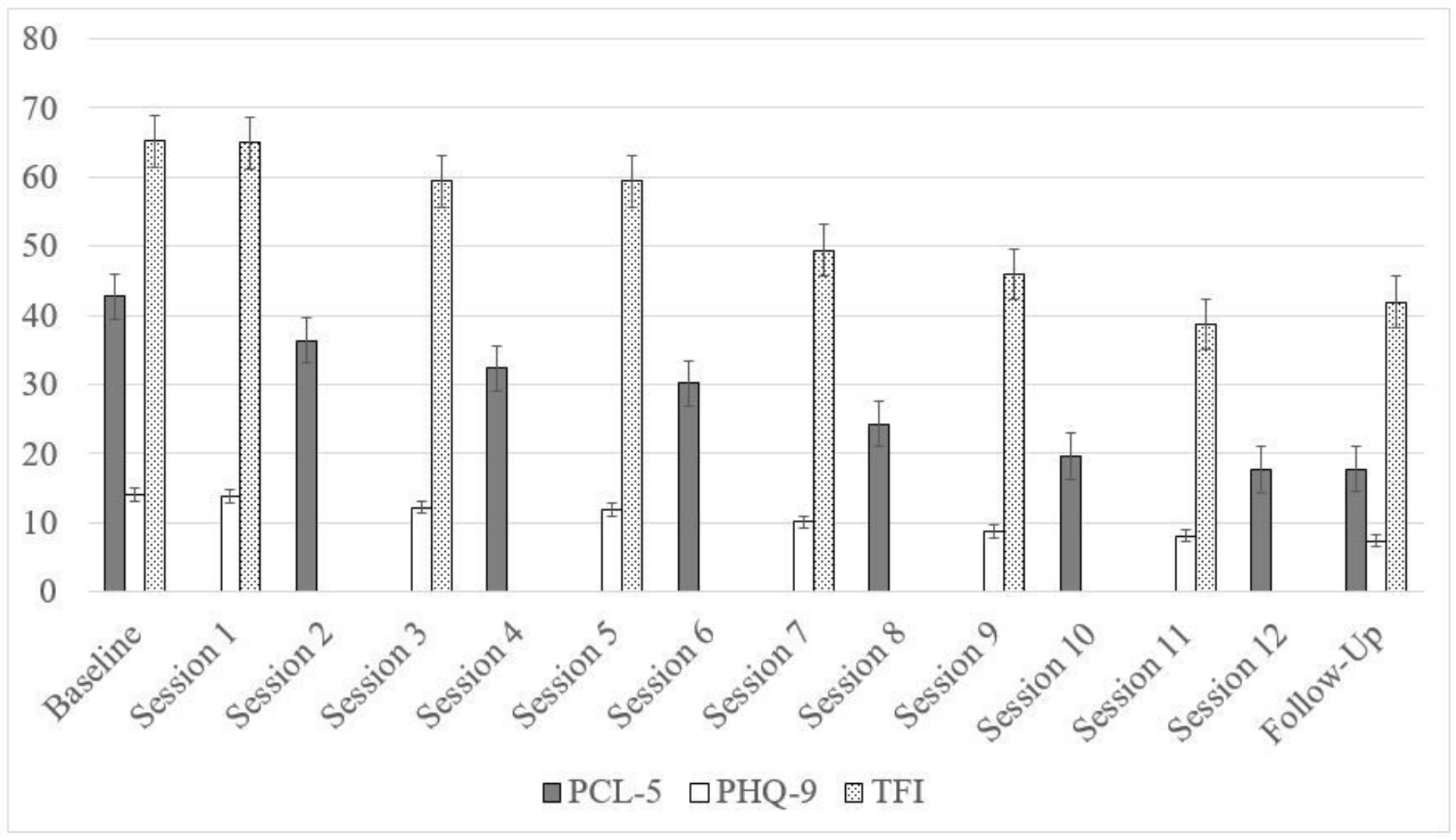

Figure 2

Session-by-Session Scores of the PCL-5, TFI, and PHQ-9 